\title{
Letter to the editor for a mistake found in a proof-of-principle study on right ventricular
}

\section{To the Editor,}

We read with great enthusiasm the article of Dr. Farag on the detection and quantification of right ventricular reversible perfusion defects by SPECT. This proof-of-principle study gave our team inspirations on further study of the right ventricle.

However, an obvious statistical or writing mistake of the $P$ value of sex (male) and age was discovered in the abstract of the article. ${ }^{1}$ As was described in Table 1 the characteristics of enrolled 34 patients, the age between the two group was $65 \pm 20$ and $63 \pm 18$, the $P$ value given in the abstract was less than 0.05 . We calculated using Student's $T$ test, the $P$ value was 0.506 . We do the same calculation to the sex (male) difference using Fisher's exact test, the $P$ value was 0.335 . Both of the two values are much more than the cutoff of 0.05 , which means differences between the two groups are not statistically significant.

Due to the fact that age and sex are all baseline characteristics, we here are wondering whether the mistakes were statistically or just a slip of the pen. Although the result will not exert any influence on the conclusion which inspired us to firmly explore the right part of the heart.

\author{
Chongchong Zhou, MS, ${ }^{a}$ \\ Jianzhou Shi, MS, ${ }^{a}$ \\ Yихіи Liu, $M S^{b}$ \\ ${ }^{a}$ Nanjing Medical University, Nanjing, Jiangsu, China \\ ${ }^{b}$ Department of Medical Statistics, Jinling Hospital, No. 305, East \\ Zhongshan Road, Nanjing 210002, Jiangsu, China \\ liu_yихіu@163.com
}

\section{Disclosures}

The authors Chongchong Zhou, Jianzhou Shi and Yuxiu Liu declare that they have no commercial or associative interest that represents a conflict of interest in connection with the work submitted.

\section{Reference}

1. Farag AA, et al. Detection and quantitation of right ventricular reversible perfusion defects by stress SPECT myocardial perfusion imaging: A proof-of-principle study. J Nucl Cardiol. 2017. https:// doi.org/10.1007/s12350-017-0954-4.

doi:10.1007/s12350-019-01608-z

Publisher's Note Springer Nature remains neutral with regard to jurisdictional claims in published maps and institutional affiliations. 